\title{
Prevenção de isquemia hepática normotérmica durante perfusão hepática in situ com três diferentes soluções de preservação: análise experimental através de termografia por radiação infravermelha em tempo real
}

\section{Prevention of normothermic hepatic ischemia during in situ liver perfusion with three different preservation solutions: experimental analysis by realtime infrared radiation thermography}

Jorge Eduardo Fouto Matias, ACBC-PR; Felipe Augusto Morais²; Daniel Massamatsu Pianovski Kato²; Vanessa Koziak²; Marcos Leal Brioschi3 ; Elizabeth Milla Tambara ${ }^{1}$; Miguel Ângelo Agulham¹; Júlio César Uili Coelho, TCBC-PR ${ }^{1}$

R E S U M O

\begin{abstract}
Objetivo: Determinar a aplicabilidade da termografia por radiação infravermelha no monitoramento da perfusão hepática in situ com diferentes soluções de preservação. Métodos: 24 ratos Wistar machos adultos, foram distribuidos aleatoriamente em quatro grupos de seis animais: grupo EC; ${ }^{\circledR}$ grupo CUST; ${ }^{\circledR}$ grupo CEL e grupo RL. Todas as soluções estavam resfriadas à exatamente $4^{\circ} \mathrm{C}$ e posicionadas $20 \mathrm{~cm}$ acima do nível do fígado. As imagens infravermelhas, com as respectivas avaliações de temperatura da superfície hepática, foram captadas em tempo real : imediatamente após a laparotomia; após a canulação dos vasos e imediatamente antes da infusão; a cada minuto cronometrado após início da infusão até o quinto minuto de infusão. As médias de temperaturas de cada momento foram comparadas intra e intergrupos através do teste da Diferença entre médias de distribuição normal, com nível de significância p 0,05. Resultados: Houve diferença entre as temperaturas do momento da laparotomia e imediatamente após a canulação; entre este último e após o primeiro minuto de perfusão; e entre as medidas ao final do primeiro e do quinto minutos de perfusão em todos os grupos. O grupo CEL mostrou diferença significativa adicional entre as temperaturas medidas ao final do primeiro e do segundo minutos. Conclusão: Foi possível avaliar o resfriamento hepático durante a perfusão das soluções de preservação utilizando-se a radiação infravermelha. As soluções tiveram comportamento semelhante entre si, com a solução Celsior mostrando potencial adicional de resfriamento significativo até ao final do segundo minuto.
\end{abstract}

Descritores: Isquemia/reperfusão. Radiação infravermelha. Preservação hepática.

\section{INTRODUÇÃO}

\begin{abstract}
A utilização de um órgão viável, que não tenha sofrido lesão isquêmica importante antes do implante no receptor, é o fator isolado mais importante na determinação do sucesso de um transplante hepático alogênico'. A prevenção de fenômenos isquêmicos evitáveis, capazes de influenciar a função primária do fígado após implante, se inicia nos cuidados intensivos com potenciais doadores de órgãos, antes mesmo do diagnóstico confirmado de morte encefálica e da autorização familiar para a retirada ${ }^{2}$. É durante a operação para retirada de órgãos que se prepara o fígado para o período em que estará desconectado de qualquer suprimento sanguíneo, até que seja implantado utilizando-se a vascularização venosa e arterial do receptor. Os fundamentos primordiais que norteiam a preserva-
\end{abstract}

ção dos órgãos neste período, prévio ao implante, são a hipotermia e a preservação da integridade celular através da infusão de soluções especificamente compostas para fornecer substratos necessários à estabilidade de membranas celulares, à posterior produção de energia e à conservação das concentrações intra e extracelulares de eletrólitos, entre outras funções ${ }^{3}$.

Com base nisso, admite-se que os períodos de isquemia "quente" (normotérmica) - 1- período imediatamente após o clampeamento da aorta no doador, durante a infusão da solução de preservação in situ antes do completo resfriamento do fígado e retirada deste no doador; 2período durante o transplante no receptor, quando se preparam as anastomoses vasculares para a reperfusão - são etapas críticas durante as quais deve-se empreender esforços para evitar danos celulares irreversíveis ao enxerto.

Trabalho realizado no Laboratório de Cirurgia Experimental do Programa de Pós-Graduação em Clínica Cirúrgica da UFPR.

1. Professor do Departamento de Cirurgia da Universidade Federal do Paraná (UFPR - PR-BR; 2. Aluno de Graduação em Medicina da UFPR Programa de Iniciação Científica; 3. Professor Colaborador do Programa de Pós-Graduação em Clínica Cirúrgica da UFPR-PR-BR. 
Apesar do segundo período referido acima ser alvo de estudos e monitoramento freqüentes, ele é inevitável, mas deve ser encurtado ao máximo através do aprimoramento técnico e manutenção da hipotermia do enxerto até o momento de reperfundí-lo com sangue venoso e arterial do receptor.

Já com respeito ao primeiro período referido, muito poucos dados disponíveis existem, pela não monitorização rigorosa do período de resfriamento dos órgãos, devido a falta de metodologia adequada, precisa e amplamente disponível em todos os hospitais onde se praticam operações de retirada de órgãos para transplante.

Teoricamente, quanto mais rápido se der o resfriamento do fígado a partir da infusão da solução de preservação à baixas temperaturas, menos oportunidades de se implantarem lesões isquêmicas nesta fase ${ }^{3}$.

A termografia por radiação infravermelha vem assumindo, em nível clínico e experimental, papel cada vez mais relevante como método seguro, não invasivo e capaz de análise do estado de perfusão dos tecidos orgânicos em várias e distintas situações ${ }^{4-9}$.

Evidencia-se no método termográfico por radiação infravermelha em tempo real uma ferramenta capaz de colaborar no monitoramento mais preciso da indução à hipotermia tecidual hepática durante sua retirada para utilização em transplantes.

Neste sentido, frente à metodologia inovadora proposta, mesmo as soluções de preservação já utilizadas para conservação hipotérmica de órgãos para transplantes, merecem atenção e avaliação de sua performance durante o período de resfriamento in situ do parênquima hepático.

O presente estudo teve como objetivo determinar a aplicabilidade da termografia por radiação infravermelha no monitoramento da perfusão hepática in situ com diferentes soluções de preservação.

\section{MÉTODOS}

O projeto de pesquisa do presente estudo foi realizado de acordo com as normas éticas estabelecidas pelo Colégio Brasileiro de Experimentação Animal (COBEA) e Lei Federal 6638, e aprovado por comitê institucional de ética em pesquisa animal.

O experimento foi realizado no Laboratório de Cirurgia Experimental do Programa de Pós-Graduação em Clínica Cirúrgica da UFPR, onde durante todo o período de estudo os animais permaneceram em ambiente com controle de temperatura $\left(22 \pm 1^{\circ} \mathrm{C}\right)$, ruído e luminosidade (ciclos claro-escuro de 12 horas - Cronomat $^{\circledR}$, Mallory do Brasil, São Paulo, SP, Brasil), agrupados em número máximo de seis, em gaiolas coletivas de aproximadamente 0,022 $\mathrm{m}^{3}(41,4 \mathrm{~cm} \times 34,4 \mathrm{~cm} \times 16 \mathrm{~cm})$. As gaiolas foram identificadas e acomodadas sobre estantes horizontais. Durante todo o período de aclimatação (14 dias), e de estudo, os animais receberam água e ração balanceada ad libitum, padrão Nuvilab $C R 1^{\circledR}$, produzido pela empresa Nuvital Nutrientes, Colombo, PR, Brasil.

\section{Animais}

Foram utilizados 24 ratos Wistar (Rattus norvegicus albinus, Roentia mammalia) adultos, machos, provenientes do biotério de criação do Setor de Ciências Biológicas da Universidade Federal do Paraná (UFPR), pesando em média 385,31g, variando entre $318 \mathrm{~g}$ e 476,4g. Os animais foram pesados e divididos aleatoriamente em 4 grupos de 06 ratos:

Grupo CEL: Seis animais com perfusão hepática in situ da solução de preservação Celsior ${ }^{\circledR}$ à temperatura de $04^{\circ}$ Celsius.

Grupo CUST: Seis animais com perfusão hepática in situ da solução de preservação Custodio $^{\circledR}$ à temperatura de $04^{\circ}$ Celsius.

Grupo EC: Seis animais com perfusão hepática in situ da solução de preservação Euro-Collins ${ }^{\circledR}$ à temperatura de $04^{\circ}$ Celsius.

Grupo RL:Seis animais com perfusão hepática in situ da solução de Ringer Lactato à temperatura de $04^{\circ}$ Celsius.

\section{Procedimento Operatório}

Sob anestesia inalatória com éter, todos os animais do estudo foram submetidos à tricotomia e anti-sepsia da região abdominal, laparotomia transversa supra-umbilical com exposição completa do fígado (Figura 1).

Após heparinização sistêmica com heparina sódica (5.000Ul/mL) na dose de $500 \mathrm{UI} / \mathrm{Kg}$ através de punção da veia cava inferior infra-hepáticas, a veia porta foi canulada com cateter Surflash IV catheter ${ }^{\circledR}-18 G$ x 1 1/ $4^{\prime \prime \prime}$, com infusão subseqüente de uma das soluções em estudo resfriadas à exatamente 4 graus centígrados positivos, e

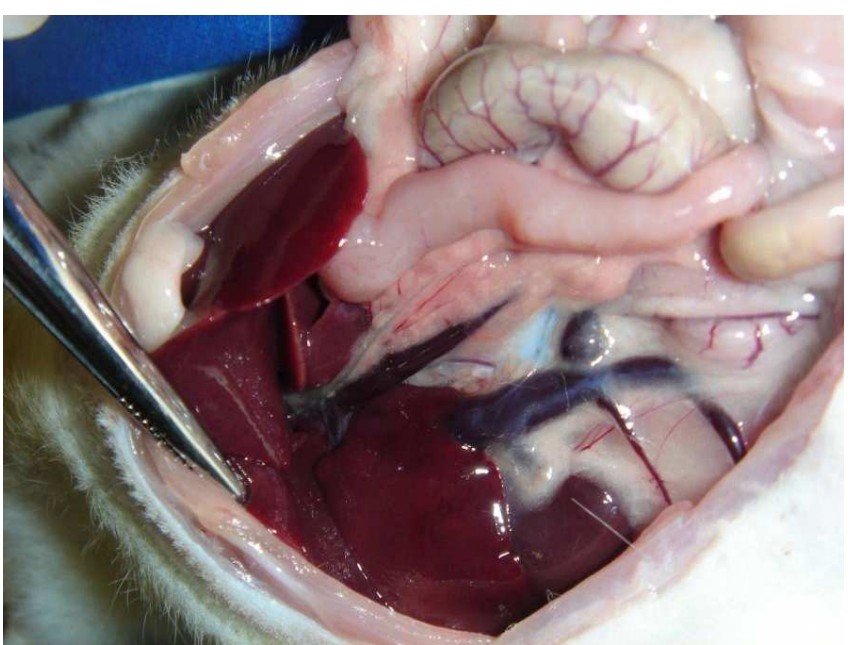

Figura 1 - Exposição hepática prévia à heparinização pela veia Cava (inferior) e à canulação da veia Porta (acima e central). 
posicionada $20 \mathrm{~cm}$ acima do nível do órgão. Para escoamento da solução após passagem pelo fígado optou-se pela abertura da veia cava inferior supra-hepática (segmento supra diafragmático) para que não interferissem nas leituras de temperatura das superfícies lobares hepáticas.

\section{Termografia Infravermelha}

As imagens infravermelhas foram captadas em tempo real por câmera Therma CAM SC $500^{\circledR}$ instalada à distância constante da superfície diafragmática dos lobos hepáticos mediano e lateral esquerdo do animal. Registradas as imagens do parênquima hepático em cada um dos momentos: 1- imediatamente após a laparotomia $\left(T_{1}\right)$; 2- após a canulação da veia porta e imediatamente antes do início da infusão da solução de preservação $\left(T_{2}\right)$; 3- após cada um dos cinco minutos cronometrados subsequentes ao início da infusão das soluções $\left(T_{3}\right.$ a $\left.T_{7}\right)$, determinando-se a temperatura em três pontos pré-determinados do parênquima sob superfície diafragmática dos lobos hepáticos mediano e lateral esquerdo (Figura 2 e 3).

Os termogramas foram analisados pelo software Therma CAM 200 Professional para gerar correspondentes numéricos às cores das imagens obtidas, para posterior confecção de gráficos e análise estatística.

\section{Análise Estatística}

Todos os dados foram tabulados em planilha do programa Microsoft Office Exce ${ }^{\circledR}$ (Microsoft Corporation, Redmond, WA, EUA), obtendo-se os valores médios (M), desvio padrão (DP) para cada variável nos diferentes tempos estudados.

Em cada um dos grupos, para cada animal, foi realizada a média aritmética das três temperaturas registradas, para cada um dos tempos. Em seguida, foi calculada a média das médias dos seis ratos de cada grupo. Através do teste estatístico de diferença entre médias com distribuição normal, foram comparadas, dentro de cada grupo de estudo, as médias finais, de cada tempo com o tempo subseqüente $\left(T_{1} \times T_{2} ; T_{2} \times T_{3} ; T_{3} \times T_{4} ; T_{4} \times T_{5} ; T_{5} \times T_{6}\right.$ e $\left.T_{6} \times T_{7}\right)$ e as após o primeiro e quinto minuto de perfusão $\left(T_{3} \times T_{7}\right)$. Para as comparações intergrupos utilizaram-se as médias das diferenças de temperatura obtidas de cada grupo ao final do primeiro minuto de infusão $\left(T_{2}-T_{3}\right)$ e ao final de todo o período de infusão - cinco minutos - $\left(T_{2}-T_{7}\right)$.

Para toda a análise estatística o nível de significância adotado foi de 5\% $(p=0,05)$.

\section{RESULTADOS}

Através da termografia por radiação infravermelha obtiveram-se as temperaturas das superfícies hepáticas de todos os animais com todas as soluções em estudo e então as médias das temperaturas de cada momento (T1 a T7), que podem ser visualizadas na tabela 1.

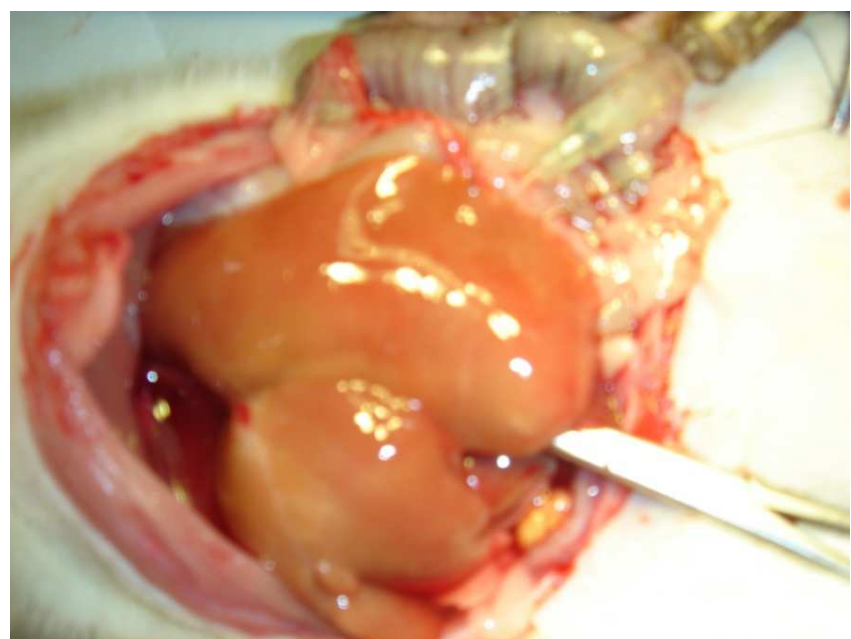

Figura 2 - Momento durante perfusão hepática: Lobos hepáticos mediano (acima) e lateral esquerdo (abaixo). Hemostática (abaixo) clampeando veia cava inferior infra-hepática e cânula (acima) inserida na veia porta.

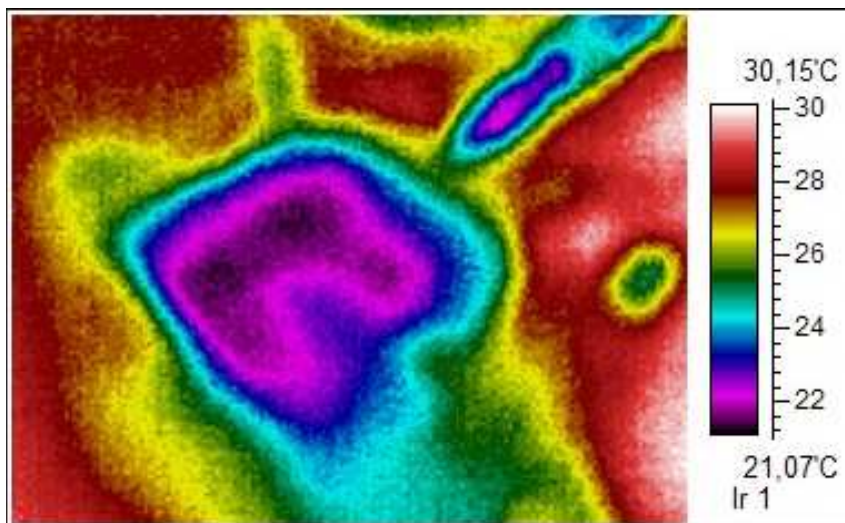

Figura 3 - Termograma de imagem infravermelha do mesmo momento de perfusão da figura 2: imagem central mais escura representando os lobos hepáticos perfundidos. À direita - escala de correspondência entre cores e temperatura.

Nota-se do quadro de medidas da tabela 1 o efeito da abertura da cavidade peritoneal sob a temperatura na superfície hepática monitorada pela imagem infravermelha, com queda de pelo menos 1 grau centígrado em todos os grupos de estudo (intervalo $T_{1}-T_{2}$ ). Como esperado, a queda mais acentuada para todos os grupos, foi no intervalo $T_{2}-T_{3}$ que corresponde ao primeiro minuto cronometrado de infusão, após canulação da veia porta. Com base nesses dados, apresenta-se um gráfico da cronologia e intensidade de resfriamento do parênquima hepático para as soluções em estudo (Figura 4).

Quanto à análise dos dados dentro de cada grupo (comparação intra-grupo), houve queda estatisticamente significativa da temperatura de forma similar em todos os grupos nos seguintes casos: 1) entre o momento da laparotomia $\left(T_{1}\right)$ e imediatamente após a canulação da veia porta $\left(T_{2}\right) ; 2$ ) entre a canulação e o final do primeiro 
Tabela 1 - Médias de temperaturas obtidas por imagem infravermelha em todos os momentos de cada grupo de estudo.

\begin{tabular}{lcccc}
\hline & Grupo EC & Grupo CUST & Grupo CEL & Grupo RL \\
\hline Média $\mathrm{T}_{1}$ & $32,87^{\circ} \mathrm{C}$ & $33,15^{\circ} \mathrm{C}$ & $33,35^{\circ} \mathrm{C}$ & $33,51^{\circ} \mathrm{C}$ \\
Média $\mathrm{T}_{2}$ & $30,74^{\circ} \mathrm{C}$ & $31,58^{\circ} \mathrm{C}$ & $32,13^{\circ} \mathrm{C}$ & $32,18^{\circ} \mathrm{C}$ \\
${\text { Média } \mathrm{T}_{3}}_{\text {Média } \mathrm{T}_{4}}^{26,52^{\circ} \mathrm{C}}$ & $24,06^{\circ} \mathrm{C}$ & $23,29^{\circ} \mathrm{C}$ & $22,37^{\circ} \mathrm{C}$ \\
${\text { Média } \mathrm{T}_{5}}_{\text {Média } \mathrm{T}_{6}}$ & $25,26^{\circ} \mathrm{C}$ & $22,66^{\circ} \mathrm{C}$ & $21,95^{\circ} \mathrm{C}$ & $21,56^{\circ} \mathrm{C}$ \\
Média $\mathrm{T}_{7}$ & $24,25^{\circ} \mathrm{C}$ & $21,85^{\circ} \mathrm{C}$ & $21,47^{\circ} \mathrm{C}$ & $20,7^{\circ} \mathrm{C}$ \\
\hline
\end{tabular}

minuto de perfusão $\left(T_{3}\right)$; e 3 ) entre o primeiro e o quinto minutos de perfusão $\left(\mathrm{T}_{3} \times \mathrm{T}_{7}\right)$.

O grupo CEL mostrou diferença significativa adicional entre as temperaturas medidas ao final do primeiro $\left(T_{3}\right)$ e do segundo minuto $\left(T_{4}\right)$ de perfusão, $\operatorname{com} p=0,0323$.

Nas comparações entre os grupos de diferentes soluções de preservação (comparação inter-grupos), houve significante perda de poder de resfriamento da solução Euro-Collins quando comparada com todas as demais estudadas (Figura 5).

\section{DISCUSSÃO}

Entre os fatores envolvidos no sucesso dos transplantes hepáticos encontram-se as soluções de preservação, que evitam a isquemia normotérmica prevenindo edema celular, dano causado por radicais livres, e depleção energética, o que resultaria em lesões irreversíveis com consequente perda funcional do orgão ${ }^{2,10}$.

No século passado, nas décadas de 80 e 90, a atividade de transplante hepático sofreu um enorme incremento a nível mundial. Neste período a solução de preservação desenvolvida na Universidade de Wisconsin (Solução de Belzer ou Viaspan ${ }^{\circledR}$ ) se tornou o padrão ouro para transplantes de fígado e outros órgãos intra-abdominais ${ }^{11,12}$. Infelizmente no presente estudo, devido a dificuldades de importação, não foi possível incluir um grupo Viaspan ${ }^{\circledR}$, uma vez que a maioria dos grupos transplantadores já substituiu esta solução por outra das do tipo HTK (soluções baseadas em Histidina, Triptofano e Cetoglutarato) ${ }^{10}$.

Entretanto, o estudo de tal solução com a metodologia proposta neste estudo (imagem infravermelha) seria importante e útil na comparação com as soluções do tipo HTK, uma vez que a solução de Belzer (Viaspan ${ }^{\circledR}$ ) possui viscosidade muito acentuada, característica alegada como fator relevante no maior número de complicações biliares pós-transplante ${ }^{13,14}$ quando se usa Viaspan ${ }^{\circledR}$ comparado com soluções muito menos viscosas (tipo HTK), capazes de maior penetração no sistema de pequenos capilares das vias biliares ${ }^{15,16}$.

No presente estudo todas as soluções empregadas foram capazes de reduzir a temperatura da superfície
Temperatura Hepática por Termografia Infravermelha durante Perfusão In Situ

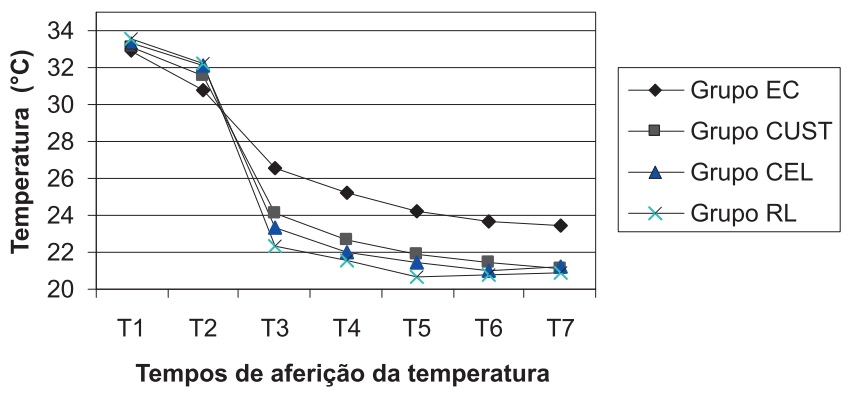

Figura 4 - Temperatura hepática por termografia infravermelha durante perfusão in situ.

\section{Comparações Intergrupos no Primeiro Minuto \\ (T2-T3) e no Período Total de Infusão (T2-T7)}

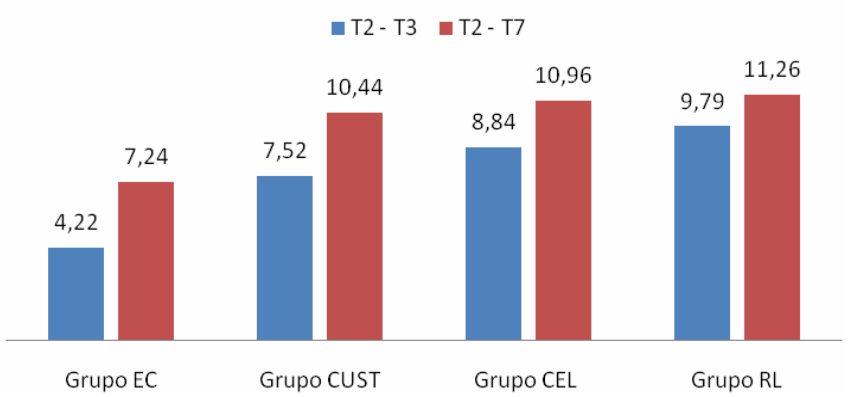

Figura 5 - Diferenças das médias de temperaturas ao final do primeiro minuto (T2-T3) e ao final do tempo total de infusão (T2-T7). Dados expressos em ${ }^{\circ} \mathrm{C}$. EC=EuroCollins; CUST=Custodiol; CEL=Celsior; RL=RingerLactato.

hepática monitorada por termografia infravermelha em momentos específicos do experimento. As temperaturas finais entretanto, podem não representar temperaturas de um órgão pronto para armazenamento em gelo para transporte. Dois aspectos do modelo empregado justificam tal achado: a perfusão realizada foi apenas in situ sem retirada posterior do órgão para perfusão adicional, como se executa nas retiradas de fígado para transplante em humanos; além disso, durante a perfusão de soluções de pre- 
servação à $4^{\circ} \mathrm{C}$ pela veia porta, não houve a interrupção do fluxo sanguíneo pela artéria hepática do animal devido ao calibre reduzido e para não haver lesão da veia porta que seria canulada.

Das soluções avaliadas neste estudo, a classicamente disponível há longo tempo é a Euro-Collins ${ }^{\circledR}$, a qual apresenta natureza intracelular e alta concentração de potássio. Estudos demonstram que altos níveis de potássio resultam em vasoconstrição, devido a abertura dos canais de cálcio presentes nas células musculares lisas do endotélio, e aumento intracelular de potássio causando lesão celular direta ${ }^{17}$. Devido a estas e outras desvantagens apresentadas pelo Euro-Collins $®$, soluções diferentes desta vêm sendo estudadas.

Curiosamente, em nosso estudo a solução EuroCollins ${ }^{\circledR}$, apesar de não possuir viscosidade similar à solução Viaspan ${ }^{\circledR}$, apresentou temperaturas aferidas significativamente superiores em cada momento de leitura a partir da infusão (Figura 4). Apesar de descrever uma curva descendente idêntica às das outras soluções em estudo, seu poder de resfriamento do parênquima hepático ficou significativamente aquém do poder de resfriamento das outras soluções em geral, inclusive da solução de Ringer-Lactato, solução com composição química imprópria para preservação de órgãos, utilizada neste estudo com fins apenas comparativos.

Custodio|® ou solução tipo HTK, foi originalmente usada na conservação do coração, e atualmente é utilizada na conservação de múltiplos órgãos. Seu uso em transplante hepático teve início na Europa no final da década de 80, onde foi demonstrada sua eficácia e segurança quando comparado ao Viaspan $®$, todavia seu uso nos Estados Unidos ocorreu somente em 2002. Entre suas principais características destacam-se menores níveis de potássio em comparação com a solução de Wisconsin, e baixa viscosidade proporcionando maior difusão na microcirculação hepática ${ }^{10}$.

Celsior $^{\circledR}$ é uma solução de preservação relativamente nova que, apesar de inicialmente formulada para conservação do coração, pode ser utilizada para preservação de vários órgãos, inclusive o fígado. Ela combina as propriedades tampão das soluções tipo HTK e as impermeabilizantes da solução Viaspan®. Em sua composição, apresenta glutationa reduzida que, além de apresentar forte poder antioxidante, previne danos ao endotélio. A solução contém ainda manitol e lactobionato para prevenção do edema celular, e glutamato para suprimento energético, entre outros componentes como a histidina e o magnésio, os quais previnem a acidose e atuam como estabilizador de membrana, respectivamente 18,19 .

Esta solução obteve, pela análise das temperaturas aferidas por termografia infravermelha, um comportamento ligeiramente superior às demais soluções, pois foi a única em que a análise estatística isolada da queda de temperatura durante segundo minuto de infusão perma- neceu com significância estatística, de maneira similar ao que ocorreu durante o primeiro minuto de infusão para todas as soluções analisadas.

A termografia por radiação infravermelha é um método seguro, não invasivo e capaz de análise do estado de perfusão dos tecidos orgânicos em distintas situações ${ }^{7,8,9,20}$, permitindo assumir papel cada vez mais relevante.

Qualquer objeto com temperatura acima de $273^{\circ} \mathrm{C}$ emite radiação infravermelha ${ }^{20}$, sendo o corpo humano, o meio ideal de estudo por apresentar emissividade infravermelha de $98 \%$, observado no comprimento de onda entre 7,5 e $13 \mu^{8}$, isto é, infravermelho longo.

Para ser analisada, a radiação térmica captada pelo sensor é transformada em uma imagem colorida em tempo real, visualizada em um monitor. Todas as imagens são distribuições de cores (Figura 3) que indicam as regiões mais aquecidas pelas cores branca e vermelha, e as áreas mais frias pelas cores azul e negro ${ }^{20}$. A avaliação é tanto quantitativa, pela diferença de temperatura média entre áreas pré-selecionadas, quanto qualitativa pelo padrão de distribuição de temperatura em determinada região.

A perfusão de órgãos sólidos, in situ ou ex vivo, de soluções resfriadas à temperaturas inferiores à temperatura normal do órgão, visualizada através de imagens infravermelhas é condição ideal, não só para monitorarmos a adequação do resfriamento de um órgão que está sendo captado para transplante, como também poderia - através da análise de padrões de perfusão alterados - auxiliar na decisão mais precisa se determinados órgãos deveriam ser rejeitados durante sua retirada, um eterno dilema em captação de órgãos, que ainda em dias atuais, continua se baseando apenas em aspectos macroscópicos do órgão alvo da retirada.

No trabalho atual pudemos observar perfeita sobreposição de curvas térmicas (Figura 4), obtidas através de termografia infravermelha, durante a perfusão in situ do fígado de ratos com diferentes soluções de preservação de órgãos, corriqueiramente utilizadas para o processo de retirada de órgãos sólidos para transplante em humanos.

Durante a retirada do fígado para transplante, não existem métodos objetivos de análise, propostos ou em utilização rotineira, que possam testemunhar de maneira precisa da integralidade do parênquima hepático a ser reimplantado, tornando esta etapa, de certa maneira, insegura, e possibilitando uma adição de risco para disfunção primária do enxerto com suas nefastas conseqüências para o organismo receptor.

A tecnologia de radiação infravermelha pode ajudar a avaliar a perfusão de órgãos sólidos, como preliminarmente demonstrado no atual estudo.

Fazem-se necessários aprofundamentos da questão em projetos de pesquisa bem estruturados, capazes de trazer contribuições inovadoras para o âmbito deste campo de pesquisa. 


\section{A B S T R A C T}

Objective: To establish the usefulness of infrared radiation thermography on monitoring in situ liver perfusion with different preservation solutions during liver harvesting. Methods: Twenty-four adult male Wistar rats, weighing $385.31 \mathrm{~g}$ were randomly divided into four groups of six animals each according to the solution used to perfuse the liver (Euro-Collins ${ }^{\circledR}$ solution - EC group; Custodio/ ${ }^{\circledR}$ solution - CUST group; Celsio ${ }^{\circledast}$ solution - CEL group and Ringer-Lactate solution - RL group). Under inhalatory ether anesthesia, animals were submitted to upper transversal laparotomy, exposure of median and leftlateral hepatic lobes, heparin injection (500 UI/Kg) through infrahepatic vena cava, portal vein infusion through $18 \mathrm{G}$ catheter of cold $\left(4^{\circ} \mathrm{C}\right)$ solution according to the group of study. Infrared images, with respective temperature avaliations from hepatic surface, were picked up in real time by Therma CAM SC500 infrared camera positioned at constant distance from three fixed points of the diaphragmatic surface of median and left lateral lobes at the following moments regarding liver perfusion: immediately after laparotomy; after portal vein cannulation and immediately before solution infusion; at each minute from the beginning of liver perfusion during five minutes. Mean temperatures of each moment were compared intra and intergroups with the difference between means test with normal distribution, with significance level of $5 \%$ $(p=0.05)$. Results: There was statistically significant difference of means temperatures between the moment of laparotomy and immediately after cannulation; between this later and after the first minute of perfusion; and between the first and fifth minutes of infusion in all groups of study in a similar way. CEL group showed additional difference between the first and second minutes means temperatures. Intergroup comparison showed Euro-Collins solution with significant less cooling power when compared to all others solutions. Conclusion: It was possible to follow the liver cooling process during preservation solutions perfusion using infrared radiation images. Preservation solutions had similar behaviors, with Celsior ${ }^{\otimes}$ solution showing additional cooling power until the second minute of perfusion. Euro-Collins solution had less cooling power than other solutions studied.

Key words: Ischemia/reperfusion. Infrared radiation. Liver preservation.

\section{REFERENCIAS}

01. Starzl TE, Demetris AJ, Van Thiel D. Liver transplantation. N Engl J Med 1989; 321(15): 1014-22.

02. Vianna RMM. Transplante hepático. In: Coelho JCU. Aparelho digestivo - clínica e cirurgia. São Paulo: Atheneu; 2005. p. 156899 .

03. Southard JH, Belzer FO. Principles of organ preservation. In: Busutti RW, Klintmam GB. Transplantation of the liver. Philadelphia: WB Saunders Company; 1996. p. 379-85.

04. Watson JC, Gorbach AM, Pluta RM, Rak R, Heiss JD, Oldfield EH. Real-time detection of vascular occlusion and reperfusion of the brain during surgery by using infrared imaging. J Neurisurg. 2002; 96(5): 918-23.

05. de Weerd L, Mercer JB, Setså LB. Intraoperative dynamic infrared thermography and free-flap surgery. Ann Plast Surg. 2006; 57(3): 279-84.

06. Cadeddu JA, Jackman SV, Schulam PG. Laparoscopic infrared imaging. J Endourol. 2001; 15(1): 111-6.

07. Mikulska D. [Contemporary applications of infrared imaging in medical diagnostics] Ann Acad Med Stetin. 2006; 52(1): 35-9; discussion 39-40.

08. Brioschi ML, Yeng LT, Pastor EMH, Teixeira MJ. Utilização da imagem infravermelha em reumatologia. Rev Bras Reumatol. 2007; 47(1): 42-51

09. Jiang LJ, Ng EY, Yeo AC, Wu S, Pan F, You WY et al. A perspective on medical infrared imaging. J Med Eng Technol. 2005 ; 29(6): 257-67.

10. Eghtesad B, Aucejo F, Fung JJ. Preservation solutions in liver transplantation: What are the options? Liver Transpl. 2006; 12(2): 226-30.

11. Jamieson NV, Sundberg R, Lindell $S$, Laravuso $R$, Southard $J H$, Belzer FO. Successful 24-30 hour preservation of the canine liver: a preliminary report. Transplant Proc. 1988; 20: 945-7.

12. Todo S, Nery S, Yanaga K, Podesta L, Gordon R, Starzl TE. Extended preservation of human liver grafts with UW solution. JAMA. 1989; 261(5): 711-4.
13. Chan SC, Liu CL, Lo CM, Fan ST. Applicability of histinetryptophan-ketoglutarate solution in right lobe adult-to-adult live donor liver transplantation. Liver Transpl. 2004; 10(11): 141521.

14. Canelo R, Hakim NS, Ringe B. Experience with histidine tryptophan ketoglutarate versus University of Wisconsin preservation in transplantation. Int Surg 2003; 88(3): 145-51.

15. Walcher F, Marzi I, Schäfer W, Flecks U, Larsen R. Undissolved particles in UW solution cause microcirculatory disturbances after liver transplantation in the rat. Transpl Int. 1995; 8(2): 161-2.

16. Tullius SG, Filatenkow A, Horch C, Mehlitz T, Ruetzel- Sleke A, Pratschke $J$ et al. Accumulation of crystal deposits in abdominal organs following perfusion with defrosted University of Wisconsin solutions. Am J Transpl. 2002; 2(7): 627-30.

17. Divisi D, Montagna P, Jegaden O, Giusti L, Berti A, Coloni GF, Ricci C, Mikaeloff P. A comparative study of EuroCollins, low potassium University of Wisconsin and cold modified blood solutions in lung preservation in acute autotransplantations in the pig. Eur J Cardiothorac Surg. 2001; 19(3): 333-8

18. Correia E. Soluções para conservação de órgãos (II) Celsior vs Viaspan.Rev de la O.F.I.L. 2002; 2(4): 13-20.

19. Dinant S, Roseboom HJ, Levi M, van Vliet AK, van Gulik TM. Hypothermic in situ perfusion of the porcine liver using Celsior or Ringer-lactate solution. Langenbecks Arch Surg. 2009; 394(1): 143-50. Epub 2008 Mar 20.

20. Brioschi ML, Yeng LT, Teixeira MJ. Diagnóstico avançado em dor por imagem infravermelha e outras aplicações. Prática Hospitalar. 2007; 50(1): 93-8.

Recebido em 09/04/2009

Aceito para publicação em 13/06/2009

Conflito de Interesse: nenhum.

Fonte de financiamento: $\mathrm{O}$ autor Felipe Augusto Morais recebeu bolsa do CNPq, pelo Programa de Iniciação Científica da UFPR, para participação no projeto. 
Matias

Prevenção de isquemia hepática normotérmica durante perfusão hepática in situ com três diferentes soluções de preservação: análise experimental através de termografia por radiação infravermelha em tempo real

\section{Como citar este artigo:}

Matias JEF, Morais FA, Kato DMP, Koziak V, Brioschi ML, Tambara EM, Agulham MA, Coelho JCU. Prevenção de isquemia hepática normotérmica durante perfusão hepática in situ com três diferentes soluções de preservação: análise experimental através de termografia por radiação infravermelha em tempo real. Rev Col Bras Cir. [periódi- co na Internet] 2010; 37(3). Disponível em URL: http://www.scielo.br/ rcbc

Endereço para correspondência:

Jorge Eduardo Fouto Matias

E-mail: jefmatias@hotmail.com 